\title{
Monte Carlo comparison of mid-size telescope designs for the Cherenkov Telescope Array
}

\author{
T. Jogler*, M. D. Wood* and J. Dumm ${ }^{\dagger}$ (for the CTA Consortium) \\ *SLAC National Accelerator Laboratory, 2575 Sand Hill Road M/S 29, Menlo Park, CA 94025, \\ USA \\ ${ }^{\dagger}$ University of Minnesota, 116 Church St SE, Minneapolis, MN 55455, USA
}

\begin{abstract}
The Cherenkov Telescope Array (CTA) is a future very high energy gamma-ray observatory. CTA will be comprised of small-, medium- and large-size telescopes covering an energy range from tens of $\mathrm{GeV}$ to hundreds of $\mathrm{TeV}$ and will surpass existing telescopes in sensitivity by an order of magnitude. The aim of our study is to find the optimal design for the medium-size telescopes (MSTs), which will determine the sensitivity in the key energy range between a few hundred GeV to about ten $\mathrm{TeV}$. To study the effect of the telescope design parameters on the array performance, we simulated arrays of 61 MSTs with $120 \mathrm{~m}$ spacing and a variety of telescope configurations. We investigated the influence of the primary telescope characteristics including optical resolution, pixel size, and light collection area on the total array performance with a particular emphasis on telescope configurations with imaging performance similar to the proposed Davis-Cotton (DC) and Schwarzschild-Couder (SC) MST designs. We compare the performance of these telescope designs, especially the achieved gamma-ray angular resolution and differential point-source sensitivity.
\end{abstract}

Keywords: Gamma-ray telescopes, MC simulations

PACS: $95.55 . \mathrm{Ka}$,

\section{INTRODUCTION}

The Cherenkov Telescope Array (CTA) is the future next generation Imaging Atmospheric Cherenkov Telescope (IACT) observatory. CTA aims to surpass the current IACT systems like HESS, MAGIC and VERITAS by an order of magnitude in sensitivity and enlarge the observable energy range from a few tens of $\mathrm{GeV}$ to far beyond one hundred $\mathrm{TeV}$ [1]. To achieve this broad energy range and high sensitivity, CTA will be comprised of three different telescope sizes. These are denoted according to their mirror diameter into large-size telescopes, medium-size telescopes, and small-size telescopes.

In this paper we investigate the effect of the optical point-spread function and the camera pixel size on the achievable point-source sensitivity. We investigate mediumsize telescopes since they are most sensitive in the energy range where the best angular resolution is achieved and small pixels sizes are most feasible.

The main motivation for this study is to determine if the current, well-tested singlemirror design (Davis-Cotton, DC) or a new two mirror design (Schwarzschild-Couder, $\mathrm{SC}$ ) would be the best choice for the medium-size CTA telescopes. The SC telescopes can achieve much smaller optical point spread functions (PSF) but require many more read out channels and more complicated mirror designs which increase their price compared to a DC telescope with similar mirror area. We simulate idealized telescope parameters for both designs and compare their gamma-ray PSF and point-source sensitivity. 


\section{SIMULATIONS}

Gamma-ray and proton air showers were simulated with the CORSIKA Monte Carlo (MC) package [2] and the QGSJet-II hadronic interaction model [4]. Simulations were performed for an array at an elevation of $2000 \mathrm{~m}$ and geomagnetic field configuration similar to the proposed southern hemisphere sites. Showers were simulated at $20^{\circ}$ zenith angle over the energy range from $10 \mathrm{GeV}$ to $30 \mathrm{TeV}$. All simulations use the same array layout comprising 61 telescopes forming a square with $120 \mathrm{~m}$ inter-telescope spacing. Each array is composed of identical telescopes.

\section{Telescope Designs}

We simulated a range of optical PSFs and pixel sizes that bracket the imaging performance of the DC- and SC-like telescope designs. The proposed designs for the DC- and SC-MST have a $68 \%$ optical PSF containment radius $\left(\mathrm{R}_{68}\right)$ of $0.02^{\circ}-0.04^{\circ}$ and $0.04^{\circ}$ $0.1^{\circ}$ over the FoV and a pixel size $\left(\mathrm{R}_{\text {pix }}\right)$ of $0.06^{\circ}$ and $0.16^{\circ}$. We use the configurations with $\mathrm{R}_{68} / \mathrm{R}_{\text {pix }}$ of $0.02^{\circ} / 0.06^{\circ}$ and $0.08^{\circ} / 0.16^{\circ}$ as representative of configurations with DC- and SC-like imaging performance respectively. For both configurations we assume a field of view of $8^{\circ}$.

After imaging resolution, the other important characteristic of the telescope optical system is the total effective light collection area $\left(A_{\text {opt }}(\lambda)\right)$, defined as the product of the mirror area and the optical efficiencies of all telescope components in the optical path including the mirrors and photosensors. We simulated a baseline telescope configuration with an aperture of $10 \mathrm{~m}$ and a mirror area of $78.5 \mathrm{~m}^{2}$. We assume a photosensor with a peak PDE of $24 \%$ at $380 \mathrm{~nm}$ and a spectral response similar to the Hamamatsu R1398. The baseline configuration has $\left\langle A_{\text {opt }}\right\rangle=11 \mathrm{~m}^{2}$ between $250 \mathrm{~nm}$ and $700 \mathrm{~nm}$ for a Cherenkov-like spectral distribution attenuated for an emission height of $10 \mathrm{~km}$. To perform a realistic comparison of the DC and SC designs at fixed cost we consider an SC configuration with $A_{\text {opt }}$ reduced by a factor of 0.562 . This factor includes the effect of both a smaller mirror area and a slightly higher photosensor PDE which could be achieved with silicon photo-multipliers.

\section{Trigger and DAQ}

The trigger and readout electronics are not simulated in detail but modeled such that the magnitude of their effect on the array performance can be estimated. The noise in each channel is modeled as the sum of a Poisson-distributed NSB term and a Gaussian-distributed electronics noise term with an RMS of 0.1 phe per channel. The NSB amplitude in each pixel is equal to $\Delta \Omega \varepsilon \sigma_{\mathrm{NSB}}$ where $\Delta \Omega$ is the pixel solid angle, $\varepsilon=\left(A_{\text {opt }} / \tilde{A}_{\text {opt }}\right)$ is the effective light collection area relative to the baseline configuration, and $\sigma_{\mathrm{NSB}}=100$ phe $\mathrm{deg}^{-2}$ is the NSB density in the focal plane. The baseline NSB density was chosen to be representative for an extragalactic observation field and an integration gate of $10 \mathrm{~ns}$. Each telescope camera containing more than 60 phe is assumed 
to trigger, and at least two telescopes must trigger to produce an array trigger. All array triggered events are further processed.

\section{Analysis}

Reconstruction of the telescope image data into event-level parameters proceeds in three stages. First, an image cleaning is performed to select pixels with statistically significant signal amplitude. The shower trajectory is then reconstructed using a geometric analysis of the moments of the light distribution in each camera. Finally a likelihoodbased reconstruction is performed using templates for the light distribution in each telescope derived from MC simulations. In addition to the event trajectory and energy, a number of parameters useful for gamma-hadron discrimination are calculated such as the goodness-of-fit of the telescope images with respect to the image templates. Background suppression is performed with the TMVA boosted decision tree (BDT) method [3]. Energy-dependent cuts on the BDT output variable and $\theta^{2}$, the squared angular separation between the reconstructed and source directions, are optimized under the assumption of a point-like source distribution with an intensity equal to $1 \%$ of the Crab Nebula flux.

\section{RESULTS \& CONCLUSIONS}
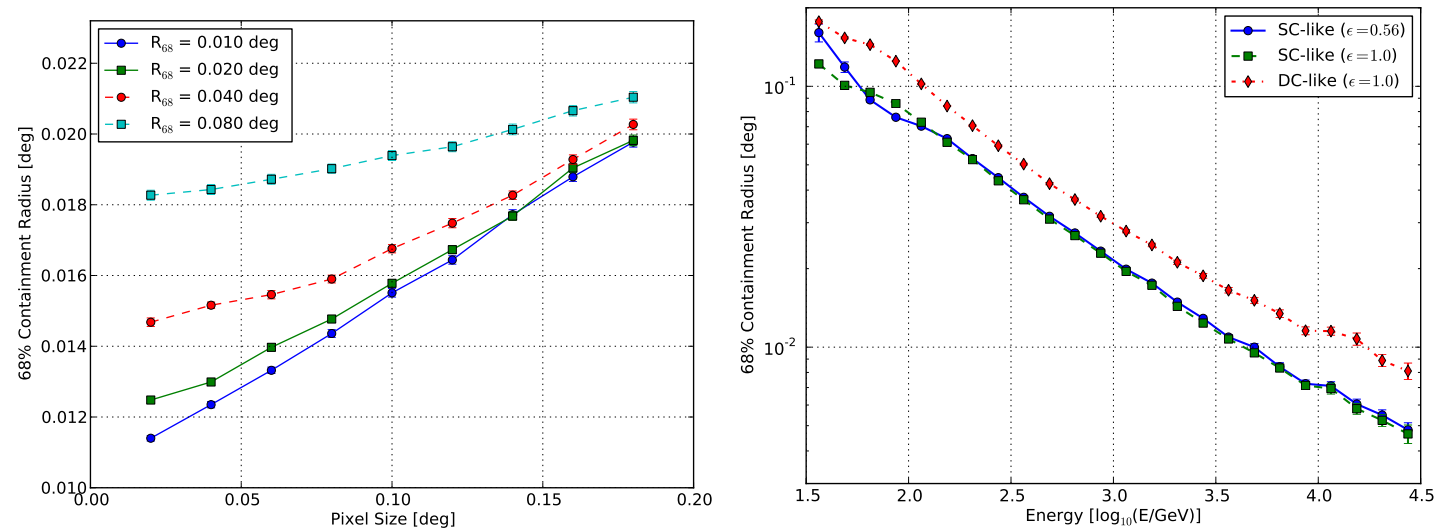

FIGURE 1. Left: $68 \%$ containment radius of the gamma-ray PSF at $1 \mathrm{TeV}$ versus pixel size shown for a 61 telescope MST array composed of telescopes with increasing 68\% optical PSF containment radii: $0.01^{\circ}$ (blue circles and solid line), $0.02^{\circ}$ (green squares with solid line), $0.04^{\circ}$ (red circles and dashed line), $0.08^{\circ}$ (cyan squares and dashed line). Right: $68 \%$ containment radius of the gamma-ray PSF versus gamma-ray energy for an array composed of telescopes with SC-like imaging performance with effective light collection area scaled by 0.56 (blue circles and solid line) and 1.0 (green squares and dashed line) and a DC-like imaging performance (red diamonds and dot-dashed line).

The gamma-ray PSF improves when reducing the pixel size as long as the optical PSF is smaller than the pixel size. The SC-like telescope array shows a $40 \%$ improved gamma-ray PSF compared to the DC-like telescopes at all energies. As shown by the comparison of SC-like arrays with $\varepsilon=1.0$ and $\varepsilon=0.56$ in Fig. 1, the gamma-ray angular 

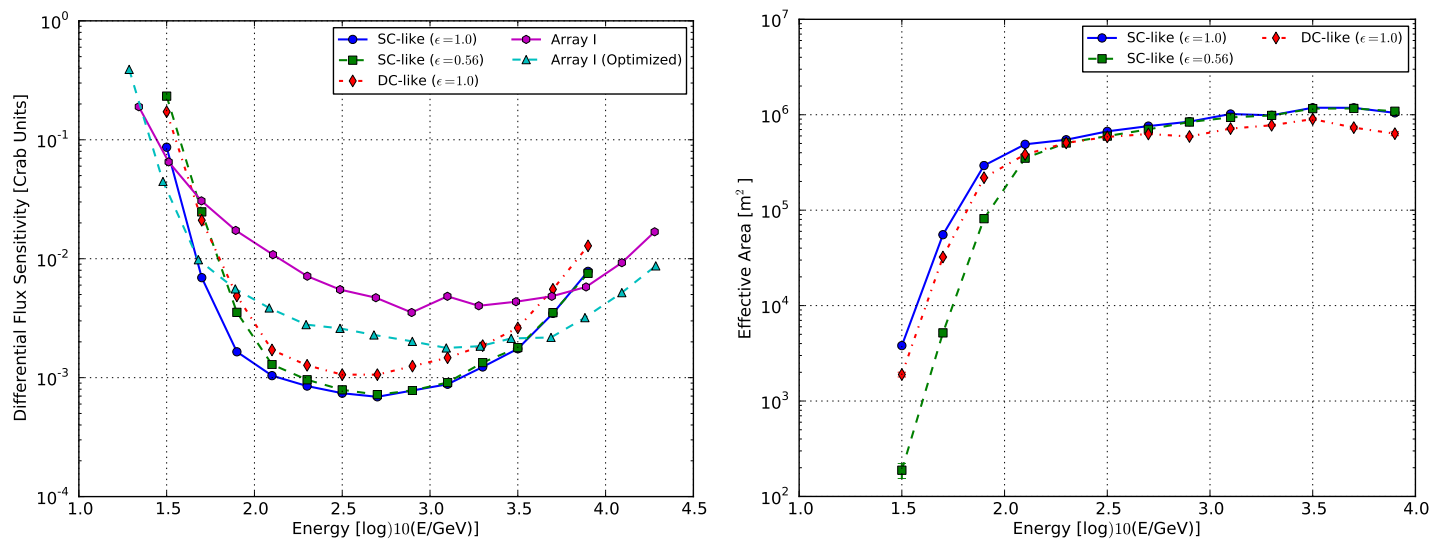

FIGURE 2. Left: Differential point-source sensitivity for a 61 telescope array with DC- (red diamonds and dot-dashed line) and SC-like (green squares and dashed line) telescope designs with assumed same cost and one of the proposed CTA designs (array I) with 18 DC-like MSTs [5] for the baseline (magenta hexagons and solid line) and optimized (cyan triangles and dashed line) analyses. The blue circles with solid line shows the performance of the SC-like telescope design when the light collection area is equal to that of the DC-like design. Right: Effective area versus gamma-ray energy for the three configurations shown in the left figure: DC-like (red diamonds and dot-dashed line), SC-like with $\varepsilon=0.56$ (green squares and dashed line), and SC-like with $\varepsilon=1.0$ (blue circles and solid line).

resolution is nearly independent of the telescope effective light collection area except at very low energies. Fig. 2 shows that the SC-like array has a $\sim 50 \%$ better differential sensitivity relative to the DC-like array at energies above $100 \mathrm{GeV}$ which is mainly due to the improved gamma-ray PSF. Below $100 \mathrm{GeV}$ the smaller light collection area of the SC-like telescope configuration is a disadvantage resulting in a higher reconstruction energy threshold and an equal or slightly worse differential sensitivity. While the SClike array is more sensitive compared to the DC-like array no SC telescope has been built to date. The results presented here provide encouragement to build an SC prototype telescope to test if the performance can be achieved under realistic conditions.

\section{ACKNOWLEDGMENTS}

We gratefully acknowledge support from the agencies and organizations listed in this page: http: //www. cta-observatory . org/ ?q=node/22

\section{REFERENCES}

1. Buckley et al.,2008, The Status and future of ground-based TeV gamma-ray astronomy. A White Paper prepared for the Division of Astrophysics of the American Physical Society,arXiv:0810.0444

2. Heck, D., Knapp, J., Capdevielle, J. N., Schatz, G., \& Thouw, T. 1998, CORSIKA: a Monte Carlo code to simulate extensive air showers.

3. Hoecker, A., Speckmayer, P., Stelzer, J., et al. 2007, arXiv:physics/0703039

4. Ostapchenko, S. 2006, Nuclear Physics B Proceedings Supplements, 151, 143

5. Bernlöhr, K, et. al., Astroparticle Physics Special Issue, in preparation. 